\title{
Soft prosthetic contact lens practice in Indian scenario
}

\author{
Biman Das, Rajeswari Mahadevan \\ Medical Research Foundation, Sankara Nethralaya, Elite School of Optometry, \\ India
}

\begin{abstract}
Aim: The aim of this study is to investigate the prescribing trend of prosthetic soft contact lens (PSCL) in a tertiary eye care center in India.
\end{abstract}

Design: Retrospective.

Methods: The electronic medical records of 223 patients who were prescribed PSCL for various indications for a period of 1 year in a tertiary eye care center were investigated. PSCL were classified to the following types: Type $A$ - black pupil with clear iris, type $B$ - no pupil with tinted iris, type $C$ - clear pupil with tinted iris; and type $D$-black pupil with tinted iris. Parameters investigated were age, gender, indication for PSCL, prescribed base curve, diameter, type and iris tint of PSCL, number of trials required to achieve desirable cosmesis or fit, duration of use of PSCL and number of PSCLs dispensed from off the shelf.

Result: A total 223 patients were prescribed PSCL for a period of 1 year, of which 221 patients were prescribed PSCL for one eye and only 2 patients for both the eyes. The mean age of the patients was 29.15 years (SD \pm 12.65$)$. Majority of the patients were male (64.57\%). Type D PSCL was prescribed for 183 eyes (81.33\%), type C PSCL for 38 eyes (16.88\%) and type $A$ PSCL for only 4 eyes (1.77\%).

Among type D PSCL users $81.96 \%$ eyes were fitted with various shades of brown colour iris tint. The main categories for prescribing type D soft PSCLs were corneal abnormalities $89.61 \%$ (164 eyes). The most common base curves prescribed among type D PSCL users were 8.6 and $8.7 \mathrm{~mm}$ and most common diameters prescribed were 13.8 and $14 \mathrm{~mm}$.

The major categories for prescribing type C PSCLs were corneal abnormalities (55.26\%; 21 eyes) and pupil or iris abnormalities (34.21\%; 13 eyes).

Only four patients (four eyes) were prescribed with type A PSCL and the only reason was leukocoria due to cataract.

In type D PSCL users, $67.21 \%$ (123 eyes) were prescribed stock lenses but for type C PSCL users, $84.21 \%$ (32 eyes) and for type A $100 \%$ (4 eyes) required customization.

Conclusions: Majority of disfigured eyes requiring PSCL are due to corneal abnormality, which can be satisfied with off the shelf PSCL. Sizeable numbers of eyes require customized PSCL for better cosmesis and fitting.

Keywords: base curve, diameter, prosthetic soft contact lens, iris tint

\section{Introduction}

Prosthesis is an artificial device to replace externally or internally a missing body part or disfigured part. One should note that prosthetic contact lens and cosmetic contact lens are not the same. Cosmetic contact lenses or decorative lenses are used to change the colour or appearance of a normal eye whereas prosthetic contact lenses are used to improve the appearance of a disfigured eye, ${ }^{1}$ or to improve

Correspondence: Biman Das

E-mail: bimandas28@gmail.com 
visual function in diseased eyes, ${ }^{2}$ and for occlusion therapy. ${ }^{3}$ Common indications for prosthetic contact lenses are albinism, amblyopia, aniridia, diplopia, fixed or dilated pupil, heterochromia, ${ }^{4}$ iridectomy, iris coloboma, photophobia, ${ }^{5}$ scarred or opacified cornea and so on. The types of prosthetic soft contact lenses (PSCLs) are determined by pupil colour and iris tint. PSCL has three zones: the central pupillary area, the tinted iris and the clear peripheral zone which is called the annulus. PSCLs are available in four types and used for different purposes: Type $A$ (black pupil with clear iris; Fig. 1a), type B (no pupil with tinted iris; Fig. 1b), type $C$ (clear pupil with tinted iris; Fig. 1c) and type D (black pupil with tinted iris; Fig. 1d). Types D and B PSCLs are usually prescribed for non-seeing or blind eyes and type A PSCL for patching therapy or for cosmetic reasons. Type C PSCL is used to improve visual function by controlling light entrance in an eye and spherical power can be incorporated in clear pupil area. In India very few manufacturers manufacture PSCLs and they are usually available in light, medium or dark brown iris tint. This is due to the fact that most of the Indian eyes have naturally brown iris colour and rarely require different colours of prosthetic iris tint. Very few studies have been published worldwide and as per our best knowledge no study has been done exclusively on prosthetic contact lens in India. The aim of this study is to understand PSCL practice better and be a quick guide to choose proper PSCL.

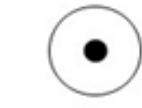

Type APSCL

a

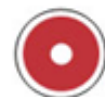

c

Type C PSCL

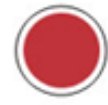

Type B PSCL

b

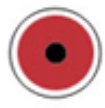

d

Type D PSCL
Fig. 1. Various types of prosthetic soft contact lenses: (a) type A; (b) type B; (c) type C; (d) type D.

\section{Materials and Methods}

For a period of 1 year (January 2013 to December 2013), 223 patients were prescribed PSCL. Data were collected retrospectively after institutional review board and ethics approval. Data were documented: age, sex, common reasons for prescribing PSCL, types of PSCL prescribed, colour of iris tint used, selection of base curve and diameter of the PSCL, number of trials required to achieve desirable cosmesis or fit, period (months) of use of prosthetic lens among old PSCL users and number of prosthetic lenses dispensed from off the shelf . PSCL were classified to 
the following types: Type A - black pupil with clear iris, type B - no pupil with tinted iris, type C - clear pupil with tinted iris; and type D - black pupil with tinted iris. Data were analyzed using Microsoft Excel 2007@. Patients who had undergone PSCL trial during the particular period but not purchased the lens due to unsuccessful fit or unwillingness for purchasing the lenses were excluded from this study.

\section{Results}

A total of 223 patients (225 eyes) were prescribed PSCL and majority were male (64.57\%). Mean age was 29.15 years (SD \pm 12.65 ) and the age range was between 6 and 78 years. Type D PSCL was prescribed for the maximum number of eyes (81.33\%) and type A PSCL for least number of eyes whereas type B PSCL was not prescribed for any eye (Fig. 2).

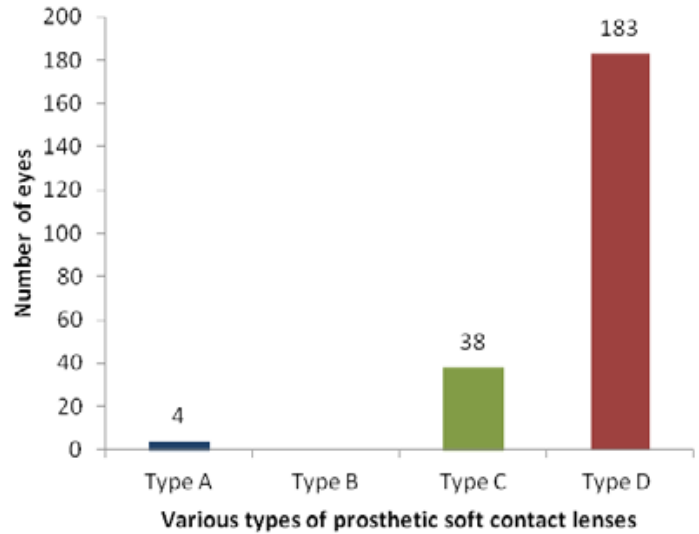

Fig. 2. Number of eyes prescribed with various types of PSCLs.

The leading causes for prescribing prosthetic contact lenses invariable of the type of lens were corneal abnormality, cataract, iris or pupillary abnormality and leukocoria. A detailed analysis was performed for each type of PSCL to gather further information.

\section{Type D PSCL}

Unilaterally, 183 patients (183 eyes) were prescribed type D PSCL and almost two-thirds were male. The categories of eye diseases for prescribing type D PSCLs were corneal abnormalities $89.61 \%$ (164 eyes), lens abnormalities $4.91 \%$ (9 eyes), pupil or iris abnormalities $3.82 \%$ (7 eyes), leukocoria $1.09 \%$ ( 2 eyes) and anterior chamber abnormalities $0.54 \%$ ( 1 eye) (Fig. 3). Further categorization of abnormalities and the number of eyes prescribed with type D PSCLs are shown in Fig. 3. 


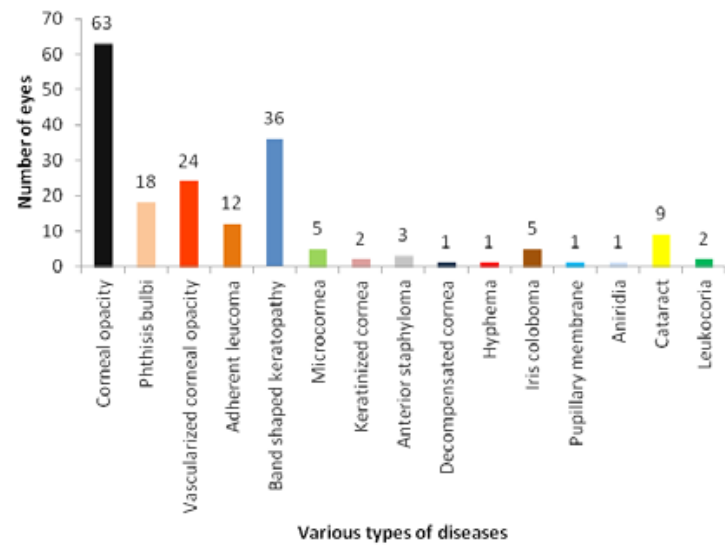

Fig. 3. Various eye diseases and number of eyes prescribed with prosthetic type $D$ contact lenses.

Tint of the prosthetic contact lens varied on the basis of the condition and the colour match with the fellow eye. The common tints used in prosthetic contact lenses for Indian eyes were light brown iris (LB), medium brown iris (MB) and dark brown iris (DB). Almost $81.96 \%$ of eyes were fitted with LB, MB or DB type D iris tint. Rest of the disfigured eyes (18.4\%) required customized iris tint for better cosmesis. The common prosthetic lens fitted had 8.6 or $8.7 \mathrm{~mm}$ base curve (BC) and 13.8 or $14.00 \mathrm{~mm}$ diameter (DIA). Few cases required modifications in BC or DIA to achieve optimal fit after trial lens evaluation. Various colours of iris tint and the number of eyes prescribed with type D PSCL are shown in Fig. 4.

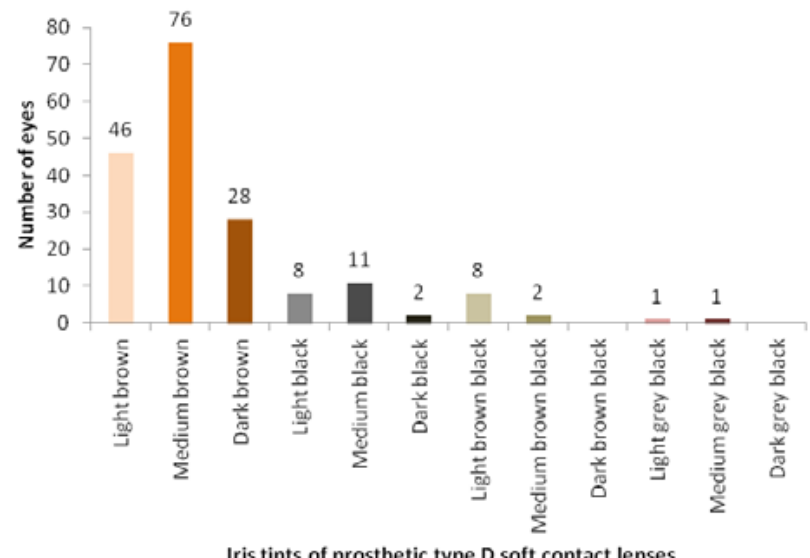

Iris tints of prosthetic type D soft contact lenses
Fig. 4. Various PSCL iris tints and the number of eyes prescribed with type D PSCL. 
Almost half of the eyes (50.8\%) were newly fitted with type D PSCL. For new cases, the average number of trials required was 1.32 (SD \pm 0.69 ; range $1-4$ times) for achieving better cosmesis and 1.36 (SD \pm 0.73 ; range 1-5 times) for desirable fit. Among the old type D PSCL users, the average wearing period was 42.70 months (SD \pm 41.70; range 2-240 months). Tables 1 and 2 show the distribution of various $\mathrm{BCs}$ and DIAs prescribed for type D PSCLs.

Table 1: Various BCs prescribed for type D prosthetic contact lenses $(n=183)$

\begin{tabular}{|l|l|}
\hline BC (in $\mathbf{~ m m})$ & Number of eyes (\%) \\
\hline 8.1 & $1(0.54)$ \\
\hline 8.3 & $7(3.82)$ \\
\hline 8.6 & $91(49.72)$ \\
\hline 8.7 & $65(35.51)$ \\
\hline 8.9 & $13(7.1)$ \\
\hline 9.0 & $1(0.54)$ \\
\hline 9.1 & $3(1.63)$ \\
\hline 9.3 & $2(1.09)$ \\
\hline
\end{tabular}

$B C$, base curve.

Table 2: Various DIAs prescribed for type D prosthetic contact lenses $(n=183)$

\begin{tabular}{|l|l|}
\hline DIA (in $\mathbf{~ m m})$ & Number of eyes (\%) \\
\hline $\mathbf{1 3 . 8}$ & $\mathbf{2 1 ( 1 1 . 4 7 )}$ \\
\hline 14.0 & $155(84.69)$ \\
\hline 14.5 & $1(0.54)$ \\
\hline 15.0 & $6(3.27)$ \\
\hline
\end{tabular}

DIA, diameter. 


\section{Type CPSCL}

Type $C$ prosthetic contact lens was prescribed for 36 patients (38 eyes) of whom 22 were male and 14 female. Only two patients were prescribed with type $C$ prosthetic contact lens for both eyes; rest was fitted unilaterally. The main categories for prescribing type C PSCLs were corneal abnormalities (55.26\%; 21 eyes), pupil or iris abnormalities (34.21\%; 13 eyes), lens abnormalities (2.63\%; 1 eye) and others (7.89\%; 3 eyes). Further categorization of abnormalities and the number of eyes prescribed with type D PSCLs are shown in Fig. 5.

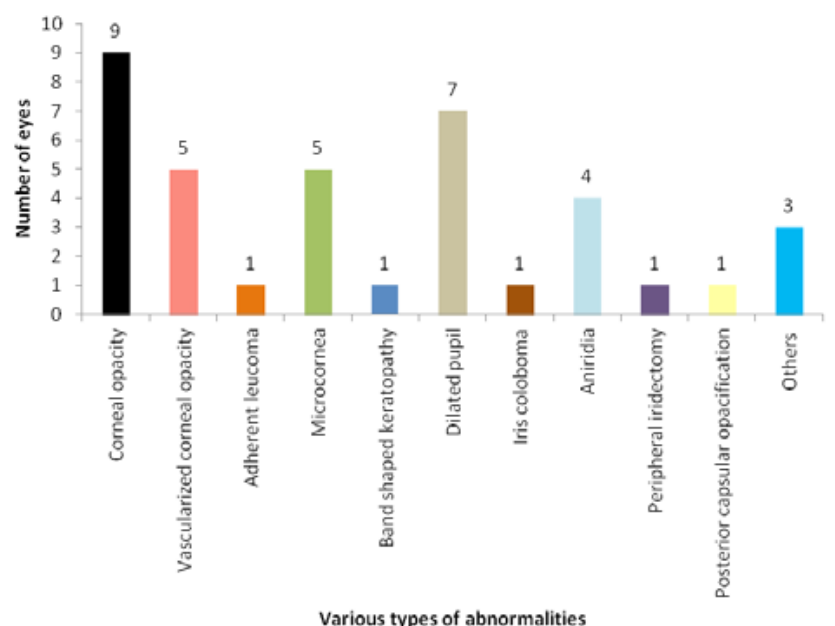

Different shades of brown colour iris tint were prescribed for all type C PSCLs and are shown in Fig. 6 along with the number of eyes prescribed with type C PSCL.

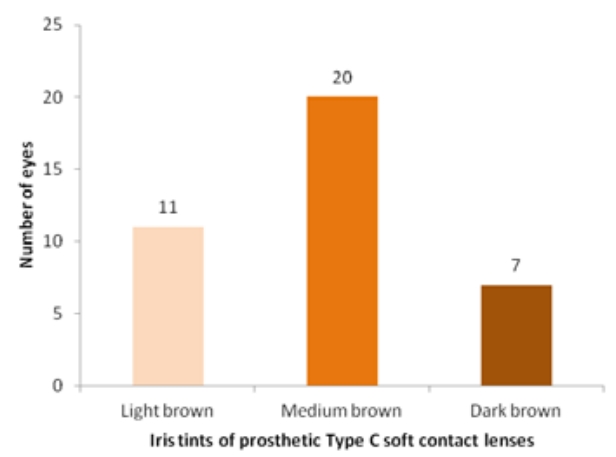

Fig. 6. Various tints prescribed for type $C$ prosthetic contact lens user.

Type C prosthetic contact lens was prescribed for 19 new patients and 17 old patients. The average number of trials required for new patients to achieve better cosmesis was 1.05 ( $S D \pm 0.22$; range 1-2 times) and to achieve best desirable fitting 
was 1.1 ( $S D \pm 0.30$; range 1-2 times). Among the old prosthetic contact lens users the average wearing period was 39.11 months (SD \pm 29.31 ; range 8-98 months).

\section{Type A PSCL}

Only four male patients (four eyes) were unilaterally prescribed type A PSCL and the only reason was cataract and all were new patients.

\section{Off the shelf vs. customized PSCL}

Among type D PSCL users, 67.21\% (123 eyes) were prescribed stock lenses and rest of them required customization for various reasons. But for type C PSCL users, $84.21 \%$ (32 eyes) customization as most of the eyes required refractive error correction along with cosmetic correction. All the type A prosthetic contact lenses needed customization because of various pupil sizes in the affected eye compared to fellow eye and the customization of pupil size was limited due to manufacturer's techniques. Table 3 shows the number of eyes for which lenses were dispensed from the stock and the number of eyes which required modified lenses.

Table 3: Off the shelf vs. customized PSCL comparisons

\begin{tabular}{|c|l|l|}
\hline Type of PSCL & $\begin{array}{l}\text { Number of eyes prescribed from } \\
\text { Stock lenses/off the shelf (\%) }\end{array}$ & $\begin{array}{l}\text { Number of eyes required } \\
\text { Customization (\%) }\end{array}$ \\
\hline A & $0(0.0)$ & $4(100)$ \\
\hline B & $0(0.0)$ & $0(0.0)$ \\
\hline C & $6(15.78)$ & $32(84.21)$ \\
\hline D & $123(67.21)$ & $60(32.78)$ \\
\hline
\end{tabular}

\section{Discussion}

Patient with disfigured eyes have limited options for cosmetic solution in India. They can wear sunglasses, undergo prosthetic shell implantation, ${ }^{6}$ and corneal tattooing, ${ }^{7}$ or wear prosthetic contact lenses for cosmetic purpose. Approximately 120,000 corneal blindness cases are reported in India by the National Programme for Control of Blindness and only 60,000 cases benefit from penetrating keratoplasty; the rest $50 \%$ vision cannot be improved due to posterior segment diseases. ${ }^{8}$ Approximately 20,000 cases of corneal blindness are added newly in each year. ${ }^{9}$ In a study, Dandona and Dandona projected a growth in the prevalence of corneal blindness mostly in unilateral cases, from $0.66 \%$ (year 2001) to $0.84 \%$ (year 2020). ${ }^{10}$ Childhood blindness and visual loss due to corneal diseases cause significant impact on the child's development, education, future jobs and quality of life. ${ }^{11}$ In India, ocular trauma, corneal ulcer, hereditary corneal dystrophy, vitamin deficiency and failed ocular surgery lead to various degrees of corneal opacity. Disfigured eyes have significant impact on a person's social life like lack of eye contact, emotional impact and withdrawal from education. Disfigured eye can 
create anxiety or poor self-image and problems in employment. ${ }^{12} \mathrm{~A}$ proper PSCL, prosthetic rigid gas permeable prosthetic contact lens, scleral prosthetic lens or enucleation of the disfigured eye and fitting of a prosthetic shell can serve as alternate options and can improve a person's self-confidence. PSCL is reasonably cheap when compared to other options. In their study, Meshel and Jessen reported that a large number of patients have disfigured eyes but only $5-10 \%$ is being treated with ocular prosthesis. ${ }^{13}$ In India uses of prosthetic contact lenses are limited and mostly soft prosthetic contact lenses are fitted in the disfigured eyes. The soft prosthetic contact lenses available in the Indian market are usually made up of high water content (67-70\%) hydrogel materials though these lenses are prescribed as conventional modality with an advisable follow-up period of 6 months to 1 year.

In most of the contact lens studies, females ${ }^{14-18}$ are the predominant contact lens users internationally as well as in India, ${ }^{19}$ but our study results showed that the predominant users of prosthetic soft contact lens are males (64.5\%); this is probably due to eye injuries as mostly males are involved in outdoor activities compared to females. ${ }^{20-22}$ Most of the disfigured eyes are fitted with brown colour prosthetic iris tint as Asians have dark brown colour iris. ${ }^{23}$ The leading cause for prescribing PSCL was corneal pathology or corneal abnormality which is due to eye injuries, complex ocular surgeries and congenital abnormalities. Our study results showed that 185 eyes (82.2\%) were fitted with PSCLs due to corneal abnormality, similar to a study done in Japan where $89.5 \%$ (40 eyes) were fitted with PSCLs due to various corneal

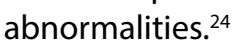

Type D PSCL prescribed majority for disfigured eyes to mask the opacity, compare to other type of PSCL and majority of them prescribed from stock lenses, probably type D PSCL does not required additional refractive error correction unlike type $C$ PSCL or not frequently required any kind of pupil size correction like type A PSCL. All the type A prosthetic contact lenses needed customization because of various pupil sizes in the affected eye compared to fellow eye and the customization of pupil size was limited due to manufacturer's techniques Additionally few disfigured eyes could not be fitted with type A prosthetic lenses due to poor cosmesis as the white opacity could not be masked from pupilary area. Such cases were fitted with type D PSCL to mask leukocoria. None of the patient prescribed occludable PSCLs for patching therapy may be it is not a popular treatment of choice in India.

Information about patients' occupation, complication with prosthetic contact lens and quality of lifestyle improvement after using PSCL is lacking in this retrospective analysis and can be planned prospectively for further study. This is a retrospective study and despite several missing information still we identified several parameters for prescribing trends in PSCLs.

\section{Conclusion}

Majority of disfigured eyes requiring PSCL are due to corneal abnormality, which can be satisfied with off the shelf PSCL. Sizeable numbers of eyes require customized PSCL for better cosmesis and fitting. 


\section{References}

1. Yildirim N, Basmak H, Sahin A. Prosthetic contact lenses: adventure or miracle. Eye Contact Lens. 2006;32(2):102-103.

2. Olali C, Mohammed M, Ahmed S, Gupta M. Contact lens for failed pupilloplasty. J Cataract Refract Surg. 2008;34(11):1995-1996.

3. Collins RS, Mcchesney ME, Mccluer CA, Schatz MP. Occlusion properties of prosthetic contact lenses for the treatment of amblyopia. J AAPOS. 2008;12(6):565-568.

4. Mcdermott ML, Chandler JW. Therapeutic uses of contact lenses. Surv Ophthalmol. 1989;33(5):381-394.

5. Rajak SN, Currie AD, Dubois VJ, Morris M, Vickers S. Tinted contact lenses as an alternative management for photophobia in stationary cone dystrophies in children. J AAPOS. 2006;10(4):336-339.

6. Raizada K, Rani D. Ocular prosthesis. Cont Lens Anterior Eye. 2007;30(3):152-162.

7. Pitz S, Jahn R, Frisch L, Duis A, Pfeiffer N. Corneal tattooing: an alternative treatment for disfiguring corneal scars. Br J Ophthalmol. 2002;86(4):397-399.

8. MoH \& FW(NPCB). Rapid assessment of Avoidable Blindness- India. Report-2006-07

9. Saini JS, Reddy MK, Jain AK, Ravindra MS, Jhaveria S, Raghuram L. Perspectives in eye banking. Indian J Ophthalmol. 1996;44(1):47-55.

10. Dandona R, Dandona L. Corneal blindness in a southern Indian population: need for health promotion strategies. Br J Ophthalmol. 2003;87(2):133-141.

11. Dandona L, Gilbert CE, Rahi JS, Rao GN. Planning to reduce childhood blindness in India. Indian J Ophthalmol. 1998;46(2):117-122.

12. Linberg JV, Tillman WT, Allara RD. Recovery after loss of an eye. Ophthal Plast Reconstr Surg. 1988;4(3):135-138.

13. Meshel LG, Jessen W. Ocular prostheses. J Am Optom Assoc. 1998;69(9):550.

14. Morgan PB, Efron N, Helland $M$, et al. Demographics of international contact lens prescribing. Cont Lens Anterior Eye. 2010;33(1):27-29.

15. Morgan PB, Efron N, Woods CA. An international survey of contact lens prescribing for presbyopia. Clin Exp Optom. 2011;94(1):87-92.

16. Tajunisah I, Ophth M, Reddy SC, Phuah SJ. Knowledge and practice of contact lens wear and care among medical students of University of Malaya. Med J Malaysia. 2008;63(3):207-210.

17. Morgan PB, Efron N. A decade of contact lens prescribing trends in the United Kingdom (19962005). Cont Lens Anterior Eye. 2006;29(2):59-68.

18. Woods CA, Jones DA, Jones LW, Morgan PB. A seven year survey of the contact lens prescribing habits of Canadian optometrists. Optom Vis Sci. 2007;84(6):505-510.

19. Thite N, Noushad B, Kunjeer G. Contact lens prescribing pattern in India--2011. Cont Lens Anterior Eye. 2013;36(4):182-185.

20. Nirmalan PK, Katz J, Tielsch JM, et al. Ocular trauma in a rural south Indian population: the Aravind Comprehensive Eye Survey. Ophthalmology. 2004;111(9):1778-1781.

21. Khan S, Maqbool A, Abdullah N, Keng MQ. Pattern of ocular injuries in stone pelters in Kashmir valley. Saudi J Ophthalmol. 2012;26(3):327-330.

22. Sinha S. Pattern of trauma to anterior segment of eyes in a tertiary eye care centre of Jharkhand. J Indian Med Assoc. 2008;106(5):289-290.

23. Obianwu HO, Rand MJ. The relationship between the mydriatic action of ephedrine and the colour of the iris. Br J Ophthalmol. 1965;49:264-270.

24. Kanemoto $M$, Toshida $H$, Takahiro I, Murakami A. Prosthetic soft contact lenses in Japan. Eye Contact Lens. 2007;33(6 Pt 1):300-303. 